doi:10.13108/2021-13-3-3

\title{
HARDY TYPE INEQUALITIES INVOLVING GRADIENT OF DISTANCE FUNCTION
}

\author{
F.G. AVKHADIEV
}

\begin{abstract}
We prove several new Hardy type inequalities in Euclidean domains; these inequalities involve the gradient of the distance function from a point to the boundary of the domain. For test functions we consider improved inequalities in form proposed by Balinsky and Evans for convex domains. Namely, in Hardy type inequalities, instead of the gradient of the test function, one takes the scalar product of the gradients of the test function and of the distance from a point to the boundary of a given domain.

In the present paper, integral Hardy type inequalities are studied in non-convex $n$ dimensional domains having a finite inradius. We prove three new Hardy type $L_{p^{-}}$ inequalities in an improved form with explicit estimates for the constants depending on the dimension of the Euclidean space $n \geqslant 2$, the inradius of the domain and two parameters $p \geqslant 1, s \geqslant n$.

Our proofs are based on three key ingredients. The first of them is related with an approximation and a special partition of the domain, in particular, we employ the approximation of the domain by subsets formed by finitely many cubes with sides parallel to the coordinate planes.

The second ingredient is the representation of the domain as a countable union of subdomains with piece-wise smooth boundaries and applying a new theorem by the author on convergence of the gradients of the distance functions for these subdomains. Moreover, we prove three new Hardy type inequalities on a finite interval, which are employed in justifying the inequalities in multi-dimensional domains.
\end{abstract}

Keywords: Hardy type inequality, inradius, gradient of distance function.

Mathematics Subject Classification: 26D10, 33C20

\section{INTRODUCTION}

Let $n \geqslant 2$ and let $\Omega \subset \mathbb{R}^{n}$ be a domain such that $\Omega \neq \mathbb{R}^{n}$. In such domains we consider weighted integral Hardy type inequalities for the functions $u \in C_{0}^{1}(\Omega)$, where $C_{0}^{1}(\Omega)$ is a family of real-valued smooth functions with compact supports in the domain $\Omega$.

We note that Hardy studied one-dimensional integral inequalities for various values of the parameters $p \in[1, \infty)$ and $s \in \mathbb{R} \backslash\{1\}$. His results can be summarized as follows, cf. [1].

Theorem 1.1. Assume that $p \in[1, \infty)$ and $s \in \mathbb{R} \backslash\{1\}$. Then for each function $f:(0, \infty) \rightarrow \mathbb{R}$ satisfying the conditions $f \in C_{0}^{1}((0, \infty))$ and $f \not \equiv 0$ the inequality holds:

$$
\int_{0}^{\infty} \frac{\left|f^{\prime}(t)\right|^{p}}{t^{s-p}} d t>\frac{|s-1|^{p}}{p^{p}} \int_{0}^{\infty} \frac{|f(t)|^{p}}{t^{s}} d t,
$$

where the constant $|s-1|^{p} / p^{p}$ is sharp.

F.G. Avkhadiev, Hardy type inequalities involving gradient of distance function.

(C) Avkhadiev F.G. 2021.

The research is supported by Russian Science Foundation (grant no. 18-11-00115).

Submitted February 5, 2021. 
In generalizations of Hardy inequalities for multi-dimensional case, a quantity $\rho(x, \partial \Omega)$ is used; this quantity is a distance from a point $x \in \Omega$ to the boundary of the domain $\Omega$, that is,

$$
\rho(x, \partial \Omega):=\inf _{y \in \mathbb{R}^{n} \backslash \Omega}|x-y| .
$$

By $\nabla \rho(x, \Omega)$ we denote the gradient of this function. We also note that hereinafter we employ the Euclidean norm $|x|=\left(x_{1}^{2}+x_{2}^{2}+\ldots+x_{n}^{2}\right)^{\frac{1}{2}}$ and the Euclidean scalar product

$$
x \cdot y=x_{1} y_{1}+x_{2} y_{2}+\ldots+x_{n} y_{n}
$$

for the vectors $x=\left(x_{1}, x_{2}, \ldots x_{n}\right) \in \mathbb{R}^{n}$ and $y=\left(y_{1}, y_{2}, \ldots, y_{n}\right) \in \mathbb{R}^{n}$.

We mention a well-known fact: $|\nabla \rho(x, \partial \Omega)|=1$ almost everywhere on $\Omega$. Therefore, in view of Cauchy-Schwarz inequality for the scalar product, for each function $u \in C_{0}^{1}(\Omega)$ we get:

$$
|\nabla u(x)| \geqslant|\nabla u(x) \cdot \nabla \rho(x, \partial \Omega)| \text { almost everywhere on } \Omega \text {. }
$$

There are many various multi-dimensional analogues of Hardy inequalities, see, for instance, books [2] and [3]. We shall be interesting in generalizations of the following two inequalities, where the parameters are chosen so that $p \in[1, \infty)$ and $s \in[1, \infty)$.

The first inequality reads as

$$
\int_{\Omega} \frac{|\nabla u(x)|^{p} d x}{\rho^{s-p}(x, \partial \Omega)} \geqslant c_{p}(s, \Omega) \int_{\Omega} \frac{|u(x)|^{p} d x}{\rho^{s}(x, \partial \Omega)} \quad \forall u \in C_{0}^{1}(\Omega),
$$

while the second inequality is the improving of the first by means of 1.1 and it reads as

$$
\int_{\Omega} \frac{|\nabla u(x) \cdot \nabla \rho(x, \partial \Omega)|^{p} d x}{\rho^{s-p}(x, \partial \Omega)} \geqslant c_{p}^{*}(s, \Omega) \int_{\Omega} \frac{|u(x)|^{p} d x}{\rho^{s}(x, \partial \Omega)} \quad \forall u \in C_{0}^{1}(\Omega) .
$$

The constants $c_{p}(s, \Omega) \in[0, \infty)$ and $c_{p}^{*}(s, \Omega) \in[0, \infty)$ are supposed to be maximal possible, that is, they are defined by the formulae

$$
c_{p}(s, \Omega)=\inf _{u \in C_{0}^{1}(\Omega), u \neq 0} \frac{\int_{\Omega} \frac{|\nabla u(x)|^{p} d x}{\rho^{s-p}(x, \partial \Omega)} d x}{\int_{\Omega} \frac{|u(x)|^{p} d x}{\rho^{s}(x, \partial \Omega)}},
$$

for inequality $(1.2)$ and

$$
c_{p}^{*}(s, \Omega)=\inf _{u \in C_{0}^{1}(\Omega), u \neq 0} \frac{\int_{\Omega} \frac{|\nabla u(x) \cdot \nabla \rho(x, \partial \Omega)|^{p} d x}{\rho^{s-p}(x, \partial \Omega)} d x}{\int_{\Omega} \frac{|u(x)|^{p} d x}{\rho^{s}(x, \partial \Omega)}}
$$

for inequality (1.3). Maximal constants $c_{p}(s, \Omega)$ and $c_{p}^{*}(s, \Omega)$ in inequalities $(1.2)$ and $(1.3)$ defined by formulae (1.4) and 1.5), respectively, are invariant with respect to linear conformal transformations of the domain. In particular, the identities

$$
c_{p}(s, \Omega)=c_{p}\left(s, k \Omega+x_{0}\right), \quad c_{p}^{*}(s, \Omega)=c_{p}^{*}\left(s, k \Omega+x_{0}\right),
$$

hold, where

$$
k \Omega+x_{0}:=\left\{y \in \mathbb{R}^{n}: y=k x+x_{0}, x \in \Omega\right\} \quad\left(x_{0} \in \mathbb{R}^{n}, k \in \mathbb{R}, k \neq 0\right) .
$$

We attract the attention of the reader to a simple but important fact. The invariancy of the inequalities with respect to scaling, that is, to transformations of form $y=k x, k>0$, yields that the constants $c_{p}(s, \Omega)$ and $c_{p}^{*}(s, \Omega)$ are dimensionless quantities. We also note that by $(1.1), 1.4$ and 1.5

$$
c_{p}^{*}(s, \Omega) \leqslant c_{p}(s, \Omega) .
$$

This paper is devoted to proving new inequalities of form (1.3) and their generalizations. There are just few results in this direction and we shall describe them below. 


\section{BASIC PROPERTIES OF FUnCtions $\rho(\cdot, \Omega)$ AND THEIR APPLICATIONS}

The distance function $\rho(\cdot, \partial \Omega)$ has numerous applications in a series of fields of theory of functions and the theory of differential elliptic equations. This function is rather well studied. Its basic properties are the following two facts, see, for instance, Chapter 2 of book [2]: the function $\rho(\cdot, \partial \Omega)$ satisfies the Lipschitz condition:

$$
|\rho(x, \partial \Omega)-\rho(y, \partial \Omega)| \leqslant|x-y| \quad \forall x, y \in \Omega,
$$

and therefore, is differentiable almost everywhere by the Rademacher theorem [4]. Thus, the distance function $\rho(\cdot, \partial \Omega)$ is differentiable on the set $\Omega \backslash S(\Omega)$, where $S(\Omega)$ is the set of all singular points, that is, all points in which $\rho(\cdot, \Omega)$ is non differentiable, and $\operatorname{mes}_{n} S(\Omega)=0$.

It is also known that $x \in \Omega \backslash S(\Omega)$ if and only if there exists a unique point $x^{\prime} \in \partial \Omega$ such that $\rho(x, \partial \Omega)=\left|x-x^{\prime}\right|$, and

$$
\nabla \rho(x, \partial \Omega)=\frac{x-x^{\prime}}{\left|x-x^{\prime}\right|}
$$

As it was mentioned in [2], this statement goes back to K.S. Motzkin [5], later it was independently obtained by other mathematicians independently of Motzkin. As an implication of (2.1) we obtain that

$$
|\nabla \rho(x, \partial \Omega)|=1 \quad \forall x \in \Omega \backslash S(\Omega) .
$$

There exist domains for which $\operatorname{mes}_{n} \bar{S}(\Omega)=0$, where $\bar{S}(\Omega)$ denotes the closure of the set $S(\Omega)$ of singular points in $\Omega$. The examples of such domains are a ball, a half-space and a convex polygon. However, it turns out that $\operatorname{mes}_{n} \bar{S}(\Omega)>0$ for a series of domains of planar and spatial domains. C. Mantegazza and A.C. Mennucci published [6] an example of a bounded convex domain $\Omega^{*} \subset \mathbb{R}^{2}$, for which the set $\bar{S}\left(\Omega^{*}\right)$ has a positive two-dimensional Lebesgue measure. In fact, in paper [6], there was give a series of such domains $\Omega^{*} \subset \mathbb{R}^{2}$. The geometric construction of $\Omega^{*}$ is based on SmithVolterra-Cantor type set on the unit circumference $\left\{x \in \mathbb{R}^{2}:|x|=1\right\}$.

The example by C. Mantegazza and A.C. Mennucci can be easily generalized for the case $n \geqslant$ 3. In particular, let $\Omega^{* *}:=\Omega^{*} \times(0, a)^{n-2}$, where $a$ is a sufficiently large positive number. Then $\operatorname{mes}_{n} \bar{S}\left(\Omega^{* *}\right)>0$. Several methods were developed for proving Hardy type inequalities in domains of form (1.2) in Euclidean domains. The simplest and most effective methods are based on employing integration by parts via Green-type formulae.

But in domains, in which $\operatorname{mes}_{n} \bar{S}(\Omega)>0$, one can not employ these simple methods for proving inequalities of form (1.3) and of their generalizations. This is why we developed a new approach based on employing representations of form

$$
\Omega=\bigcup_{j=1}^{\infty} \Omega_{j},
$$

where $\Omega_{j}$ are chosen so that $\operatorname{mes}_{n} \bar{S}\left(\Omega_{j}\right)=0$. Moreover, we shall need statements, which we proved in recent papers [7] and [8] on the convergence of the distance functions and their gradients under exhausting a domain.

Let us recall the main result of paper [8].

Theorem 2.1. Let $n \geqslant 2$ and

$$
\Omega=\bigcup_{j=1}^{\infty} \Omega_{j},
$$

where $\Omega$ and $\Omega_{j}$ are open sets in the Euclidean space $\mathbb{R}^{n}, \Omega \neq \mathbb{R}^{n}, K$ is some compact set in $\mathbb{R}^{n}$, and the conditions

$$
K \subset \Omega_{j} \subset \Omega_{j+1} \quad \forall j \in \mathbb{N}
$$

are satisfied. Then the following statements hold:

(i) $\rho\left(x, \partial \Omega_{j}\right) \rightarrow \rho(x, \partial \Omega)$ as $j \rightarrow \infty$ uniformly on the compact set $K$, that is,

$$
\lim _{j \rightarrow \infty} \max _{x \in K}\left|\rho(x, \partial \Omega)-\rho\left(x, \partial \Omega_{j}\right)\right|=0 ;
$$


(ii) there exists a set $S \subset K$ such that its $n$-dimensional Lebesgue measure satisfies the identity $\operatorname{mes}_{n} S=0$ and

$$
\lim _{j \rightarrow \infty} \nabla \rho\left(x, \partial \Omega_{j}\right)=\nabla \rho(x, \partial \Omega)
$$

at each point $x \in K \backslash S$.

In papers [7] and [8] we also obtained similar results on convergence under outer approximations of the domain and on their applications for verifying strict forms of Hardy type inequalities.

In particular, in paper [8] we proved the following theorem.

Theorem 2.2. Assume that $n \geqslant 2$ and $\Omega \subset \mathbb{R}^{n}$ is a convex domain, $\Omega \neq \mathbb{R}^{n}, p \in[1, \infty)$ and $s \in(1, \infty)$. Then

$$
c_{p}^{*}(s, \Omega)=\frac{(s-1)^{p}}{p^{p}} .
$$

A.A. Balinsky and W.D. Evans had proved this theorem earlier in paper [9] for the case $p=s \in$ $(1, \infty)$ and under some additional conditions for the domain. It was pointed out in paper [9] that some inequalities of form 1.3 have applications in problems on images recognition and recovering.

The next theorem belongs to the author, see [8] and [10].

Theorem 2.3. Let $n \geqslant 2$ and $\Omega \subset \mathbb{R}^{n}$ is an arbitrary domain satisfying the condition $\Omega \neq \mathbb{R}^{n}$. If $p \in[1, \infty)$ and $s \in[n, \infty)$, then

$$
c_{p}(s, \Omega) \geqslant c_{p}^{*}(s, \Omega) \geqslant \frac{(s-n)^{p}}{p^{p}} .
$$

These estimates are sharp in the sense that there exist both bounded and unbounded domains $\Omega \neq \mathbb{R}^{n}$, for which

$$
c_{p}(s, \Omega)=c_{p}^{*}(s, \Omega)=\frac{(s-n)^{p}}{p^{p}} .
$$

Other estimates for the constants $c_{p}(s, \Omega)$ can be found in papers [11]-[16].

\section{INEQUALITIES IN DOMAINS WITH A FINITE INRADIUS}

An inradius $\rho(\Omega)$ of a domain $\Omega$ is defined by the identity

$$
\rho(\Omega)=\sup _{x \in \Omega} \rho(x, \partial \Omega) .
$$

It is obvious that the inradius of a bounded domain is finite. We observe that the inverse is not true: there exist unbounded domains with a finite inradius.

As $p=1$ and $s \in(n, \infty)$, by 1.3 , 1.5) and Theorem 2.3 , in an arbitrary domain $\Omega \subset \mathbb{R}^{n}, \Omega \neq \mathbb{R}^{n}$, the inequality holds:

$$
\int_{\Omega} \frac{|\nabla u(x) \cdot \nabla \rho(x, \partial \Omega)| d x}{\rho^{s-1}(x, \partial \Omega)} \geqslant(s-n) \int_{\Omega} \frac{|u(x)| d x}{\rho^{s}(x, \partial \Omega)} \quad \forall u \in C_{0}^{1}(\Omega),
$$

and there exist both bounded and unbounded domains $\Omega$, for which the constant $c_{1}^{*}(s, \Omega)=s-n$ in this inequality is sharp, that is, it is maximal possible. We recall one feature of Hardy type inequalities: the sharpness of the constant does not mean the existence of an extremal function $u \not \equiv 0$, at which the inequality becomes the identity.

Let us prove that inequality (3.1) can be essentially strengthened for each domain $\Omega$ with a finite inradius $\rho(\Omega)$. Namely, we are going to prove the following two statements.

Theorem 3.1. Assume that $n \geqslant 2, s \in(n, \infty)$ and $\Omega \subset \mathbb{R}^{n}$ is an arbitrary domain obeying the only condition $\rho(\Omega)<\infty$. Then

$$
\int_{\Omega} \frac{|\nabla u(x) \cdot \nabla \rho(x, \partial \Omega)| d x}{\rho^{s-1}(x, \partial \Omega)}-(s-n) \int_{\Omega} \frac{|u(x)| d x}{\rho^{s}(x, \partial \Omega)} \geqslant S_{1}(u, \Omega) \quad \forall u \in C_{0}^{1}(\Omega),
$$

where

$$
S_{1}(u, \Omega)=\frac{s-n}{(s-1) \rho^{s}(\Omega)} \int_{\Omega}|u(x)| d x+\frac{1}{\rho^{s}(\Omega)} \int_{\Omega} \rho(x, \partial \Omega)|\nabla u(x) \cdot \nabla \rho(x, \partial \Omega)| d x .
$$


The next statement is a simultaneous generalization and implication of Theorem 3.1 .

Theorem 3.2. Assume that $n \geqslant 2$ and $\Omega \subset \mathbb{R}^{n}$ is an arbitrary domain obeying the only condition $\rho(\Omega)<\infty$. If $p \in[1, \infty)$ and $s \in(n, \infty)$, then

$$
\int_{\Omega} \frac{|\nabla u(x) \cdot \nabla \rho(x, \partial \Omega)|^{p} d x}{\rho^{s-p}(x, \partial \Omega)}-\frac{(s-n)^{p}}{p^{p}} \int_{\Omega} \frac{|u(x)|^{p} d x}{\rho^{s}(x, \partial \Omega)} \geqslant S_{p}(u, \Omega) \quad \forall u \in C_{0}^{1}(\Omega),
$$

where

$$
S_{p}(u, \Omega)=\frac{(s-n)^{p}}{p^{p-1}(s-1) \rho^{s}(\Omega)} \int_{\Omega}|u(x)|^{p} d x+\frac{1}{\rho^{p s}(\Omega)} \int_{\Omega} \frac{|\nabla u(x) \cdot \nabla \rho(x, \partial \Omega)|^{p}}{\rho^{s-p-p s}(x, \partial \Omega)} d x .
$$

As we have mentioned above, since $|\nabla \rho(x, \partial \Omega)|=1$ almost everywhere on $\Omega$, by the Cauchy-Schwarz inequality for the scalar product inequality $(1.1)$ is true. This is why Theorem 3.2 implies the following corollary.

Corollary 3.1. Assume that $n \geqslant 2, p \in[1, \infty), s \in(n, \infty)$ and $\Omega \subset \mathbb{R}^{n}$ is an arbitrary domain obeying the condition $\rho(\Omega)<\infty$. Then

$$
\int_{\Omega} \frac{|\nabla u(x)|^{p} d x}{\rho^{s-p}(x, \partial \Omega)}-\frac{(s-n)^{p}}{p^{p}} \int_{\Omega} \frac{|u(x)|^{p} d x}{\rho^{s}(x, \partial \Omega)} \geqslant S_{p}^{*}(u, \Omega) \quad \forall u \in C_{0}^{1}(\Omega),
$$

where

$$
S_{p}^{*}(u, \Omega)=\frac{(s-n)^{p}}{p^{p-1}(s-1) \rho^{s}(\Omega)} \int_{\Omega}|u(x)|^{p} d x+\frac{1}{\rho^{p s}(\Omega)} \int_{\Omega} \frac{|\nabla u(x)|^{p}}{\rho^{s-p-p s}(x, \partial \Omega)} d x .
$$

As $s=n$, non-trivial analogues of inequality (3.1) are possible via changing the weight functions with applying logarithmic factors in the way how this was done in paper [10] in a less general situation, when the inequalities involve $|\nabla u(x)|$ instead the absolute value of the scalar product $|\nabla u(x) \cdot \nabla \rho(x, \partial \Omega)|$. Namely, by applying the logarithmic factors, we are going to prove the following two theorems.

Theorem 3.3. Assume that $p \in[1, \infty), n \geqslant 2$ and $\Omega \subset \mathbb{R}^{n}$ is an arbitrary domain obeying the condition $\rho(\Omega)<\infty$. Then the inequality

$$
\int_{\Omega} \frac{|\nabla u(x) \cdot \nabla \rho(x, \partial \Omega)|^{p}}{\rho^{n-p}(x, \partial \Omega)} \ln ^{p} \frac{\rho(\Omega)}{\rho(x, \partial \Omega)} d x \geqslant \frac{1}{p^{p}} \int_{\Omega} \frac{|u(x)|^{p} d x}{\rho^{n}(x, \partial \Omega)} \quad \forall u \in C_{0}^{1}(\Omega)
$$

holds true.

We note that in the general case. the logarithmic factor in this inequality is an essential ingredient. For instance, for the domain

$$
\Omega_{1}=\left\{x \in \mathbb{R}^{n}: 0<|x|<1\right\},
$$

that is, for the unit ball with the punctured center, there exists no positive constant $c(p, n)$ such that the inequality holds:

$$
\int_{\Omega_{1}} \frac{\left|\nabla u(x) \cdot \nabla \rho\left(x, \partial \Omega_{1}\right)\right|^{p}}{\rho^{n-p}\left(x, \partial \Omega_{1}\right)} d x \geqslant c(p, n) \int_{\Omega_{1}} \frac{|u(x)|^{p} d x}{\rho^{n}\left(x, \partial \Omega_{1}\right)} \quad \forall u \in C_{0}^{1}\left(\Omega_{1}\right) .
$$

We also stress that Theorem 3.3 implies the following statement, which we proved in paper [10].

Corollary 3.2. Assume that $n \geqslant 2$ and $\Omega \subset \mathbb{R}^{n}$ is a domain obeying the condition $\rho(\Omega)<\infty$. Then the inequalities hold:

$$
\begin{aligned}
& \int_{\Omega} \frac{|\nabla u(x)|}{\rho^{n-1}(x, \partial \Omega)} \ln \frac{\rho(\Omega)}{\rho(x, \partial \Omega)} d x \geqslant \int_{\Omega} \frac{|u(x)| d x}{\rho^{n}(x, \partial \Omega)} \quad \forall u \in C_{0}^{1}(\Omega), \\
& \int_{\Omega}|\nabla u(x)|^{n} \ln ^{n} \frac{\rho(\Omega)}{\rho(x, \partial \Omega)} d x \geqslant \frac{1}{n^{n}} \int_{\Omega} \frac{|u(x)|^{n} d x}{\rho^{n}(x, \partial \Omega)} \quad \forall u \in C_{0}^{1}(\Omega) .
\end{aligned}
$$


In Theorem 3.3 , the kernel of the integral is strengthened by means of the factor

$$
\ln ^{p} \frac{\rho(\Omega)}{\rho(x, \partial \Omega)} \rightarrow \infty, \quad \text { as } \quad \rho(x, \partial \Omega) \rightarrow 0
$$

Now we consider the case, when the kernel of the integral in the right hand side of the inequality is weakened by means of a logarithmic factor.

Theorem 3.4. Assume that $p \in[1, \infty), n \geqslant 2$ and $\Omega \subset \mathbb{R}^{n}$ is a domain obeying the condition $\rho(\Omega)<\infty$. Then

$$
\int_{\Omega} \frac{|\nabla u(x) \cdot \nabla \rho(x, \partial \Omega)|}{\rho^{n-1}(x, \partial \Omega)} d x \geqslant \int_{\Omega} \frac{|u(x)|}{\rho^{n}(x, \partial \Omega)} \ln ^{-2} \frac{e \rho(\Omega)}{\rho(x, \partial \Omega)} d x \quad \forall u \in C_{0}^{1}(\Omega) .
$$

As usually, by $e \approx 2.718$ we denote the radix of the natural logarithm.

If $p>1$, then the condition $u \in C_{0}^{1}(\Omega)$ implies that $v:=|u|^{p} \in C_{0}^{1}(\Omega)$. This is why the following corollary is true.

Corollary 3.3. Assume that $p \in[1, \infty), n \geqslant 2$ and $\Omega \subset \mathbb{R}^{n}$ is an arbitrary domain obeying the condition $\rho(\Omega)<\infty$. Then

$$
\int_{\Omega} \frac{|u(x)|^{p-1}|\nabla u(x) \cdot \nabla \rho(x, \partial \Omega)|}{\rho^{n-1}(x, \partial \Omega)} d x \geqslant \frac{1}{p} \int_{\Omega} \frac{|u(x)|^{p} A(x, \Omega)}{\rho^{n}(x, \partial \Omega)} d x \quad \forall u \in C_{0}^{1}(\Omega),
$$

where

$$
A(x, \Omega)=\ln ^{-2} \frac{e \rho(\Omega)}{\rho(x, \partial \Omega)} .
$$

For proving the theorems, we shall employ the following four lemmata generalizing and strengthening corresponding statements, which we proved in [10].

Lemma 3.1. Assume that $c=$ const $>0$ and in a domain $\Omega \subset \mathbb{R}^{n}$ two continuous functions $w_{1}=w_{1}(x)>0, w_{2}=w_{2}(x)>0$ are defined and a functional $J: C_{0}^{1}(\Omega) \rightarrow \mathbb{R}$ is defined as well.

If

$$
J(u)+\int_{\Omega}|u(x)| w_{1}(x) d x \leqslant c \int_{\Omega}|\nabla u(x) \cdot \nabla \rho(x, \partial \Omega)| w_{2}(x) d x,
$$

for each real-valued function $u \in C_{0}^{1}(\Omega)$, then for each $p \in(1, \infty)$ and each real-valued function $u \in C_{0}^{1}(\Omega)$ the inequality holds:

$$
p J\left(|u|^{p}\right)+\int_{\Omega}|u(x)|^{p} w_{1}(x) d x \leqslant(c p)^{p} \int_{\Omega}|\nabla u(x) \cdot \nabla \rho(x, \partial \Omega)|^{p} w_{1}^{1-p}(x) w_{2}^{p}(x) d x .
$$

Proof. Assume that $p>1$ and $u \in C_{0}^{1}(\Omega)$ is a real-valued function. Then the function $v:=|u|^{p} \in C_{0}^{1}(\Omega)$ since

$$
\nabla v=\nabla|u|^{p}=p|u|^{p-1}(\nabla u) \operatorname{sign} p(u)
$$

and a function $|u|^{p-1} \operatorname{sign}(u)$ is continuous since $u \in C_{0}^{1}(\Omega)$ and $p>1$. By the assumption of the lemma, the function $v=|u|^{p} \in C_{0}^{1}(\Omega)$ satisfies the inequality

$$
J\left(|u|^{p}\right)+\int_{\Omega}|u(x)|^{p} w_{1}(x) d x \leqslant\left. c \int_{\Omega}|\nabla| u(x)\right|^{p} \cdot \nabla \rho(x, \partial \Omega) \mid w_{2}(x) d x .
$$

We estimate the integral in the right hand side from above by employing a simple inequality

$$
\left.\left.|\nabla| u(x)\right|^{p} \cdot \nabla \rho(x, \partial \Omega)|\leqslant p| u(x)\right|^{p-1}|\nabla u(x) \cdot \nabla \rho(x, \partial \Omega)|
$$

and the Young inequality

for the quantities

$$
a^{p-1} b \leqslant\left(1-\frac{1}{p}\right) a^{p}+\frac{b^{p}}{p}
$$

$$
a=|u(x)| w_{1}^{\frac{1}{p}}(x), \quad b=c p|\nabla u(x)| w_{1}^{\frac{1}{p}-1}(x) w_{2}(x) .
$$


We get:

$$
\begin{aligned}
J\left(|u|^{p}\right)+\int_{\Omega}|u(x)|^{p} w_{1}(x) d x \leqslant & \left(1-\frac{1}{p}\right) \int_{\Omega}|u(x)|^{p} w_{1}(x) d x \\
& +\frac{1}{p} \int_{\Omega}(c p)^{p}|\nabla u(x) \cdot \nabla \rho(x, \partial \Omega)|^{p} w_{1}^{1-p}(x) w_{2}^{p}(x) d x,
\end{aligned}
$$

and this completes the proof.

Lemma 3.2. Assume that $0<a \leqslant b, k \in\{1,2, \ldots, n\}$, where $n$ is a natural number, $n \geqslant 2$, $s \in(n, \infty)$. If $f:[0, a] \rightarrow \mathbb{R}$ is an absolutely continunous function satisfying the condition $f(0)=0$, then an inequality holds:

$$
\int_{0}^{a} \frac{|f(t)|}{t^{s-k+1}}\left(1+\frac{t^{s}}{(s-1) b^{s}}\right) d t \leqslant \frac{1}{s-n} \int_{0}^{a} \frac{\left|f^{\prime}(t)\right|}{t^{s-k}}\left(1-\frac{t^{s}}{b^{s}}\right) d t .
$$

Proof. Since

$$
|f(t)| \leqslant \int_{0}^{t}\left|f^{\prime}(\tau)\right| d \tau
$$

we get:

$$
\int_{0}^{a} \frac{|f(t)|}{t^{s-k+1}}\left(1+\frac{t^{s}}{(s-1) b^{s}}\right) d t \leqslant \int_{0}^{a} \frac{1}{t^{s-k+1}}\left(1+\frac{t^{s}}{(s-1) b^{s}}\right) d t \int_{0}^{t}\left|f^{\prime}(\tau)\right| d \tau .
$$

Swapping the integration order in the iterated integral and calculating the internal integral, we obtain:

$$
\int_{0}^{a} \frac{|f(t)|}{t^{s-k+1}}\left(1+\frac{t^{s}}{(s-1) b^{s}}\right) d t \leqslant \int_{0}^{a} \frac{\left|f^{\prime}(\tau)\right|}{\tau^{s-k}} T(\tau) d \tau
$$

where

$$
T(\tau)=\tau^{s-k} \int_{\tau}^{a} \frac{1}{t^{s-k+1}}\left(1+\frac{t^{s}}{(s-1) b^{s}}\right) d t
$$

Letting $t=x \tau$ and employing the estimates

$$
\begin{aligned}
& a \leqslant b, \quad s>n, \quad x^{k-1} \leqslant x^{n-1} \quad \text { as } \quad 1 \leqslant x \leqslant \frac{b}{\tau}, \\
& 1-y^{s-n}+\frac{s-n}{n(s-1)}\left(y^{s-n}-y^{s}\right) \leqslant 1-y^{s} \quad \text { as } \quad 0 \leqslant y=\tau / b \leqslant 1,
\end{aligned}
$$

we find:

$$
\begin{aligned}
T(\tau) & \leqslant \int_{1}^{\frac{b}{\tau}} \frac{1}{x^{s-n+1}}\left(1+\frac{x^{s} \tau^{s}}{(s-1) b^{s}}\right) d x \\
& =\frac{1-y^{s-n}}{s-n}+\frac{y^{s-n}-y^{s}}{n(s-1)} \leqslant \frac{1-y^{s}}{s-n}, \quad y=\frac{\tau}{b} .
\end{aligned}
$$

Thus, we have obtained the inequality

$$
\int_{0}^{a} \frac{|f(t)|}{t^{s-k+1}}\left(1+\frac{t^{s}}{(s-1) b^{s}}\right) d t \leqslant \frac{1}{s-n} \int_{0}^{a} \frac{\left|f^{\prime}(\tau)\right|}{\tau^{s-k}}\left(1-\frac{\tau^{s}}{b^{s}}\right) d t,
$$

and this completes the proof. 
Lemma 3.3. Assume that $0<a \leqslant b, k \in\{1,2, \ldots, n\}$, where $n$ is a natural number, $n \geqslant 2$. If $f:[0, a] \rightarrow \mathbb{R}$ is an absolutely continuous function satisfying the condition $f(0)=0$, then an inequality holds true:

$$
\int_{0}^{a} \frac{|f(t)|}{t^{n-k+1}} d t \leqslant \int_{0}^{a} \frac{\left|f^{\prime}(t)\right|}{t^{n-k}} \ln \frac{b}{t} d t
$$

Proof. Following the lines of the previous proof, we have:

$$
\int_{0}^{a} \frac{|f(t)|}{t^{n-k+1}} d t \leqslant \int_{0}^{a} \frac{1}{t^{n-k+1}} d t \int_{0}^{t}\left|f^{\prime}(\tau)\right| d \tau=\int_{0}^{a} \frac{\mid f^{\prime}(\tau)}{\tau^{n-k}} T^{*}(\tau) d \tau
$$

where

$$
T^{*}(\tau)=\tau^{n-k} \int_{\tau}^{a} \frac{d t}{t^{n-k+1}} \leqslant \tau^{n-k} \int_{\tau}^{b} \frac{d t}{t^{n-k+1}}=\int_{1}^{b / \tau} \frac{d x}{x^{n-k+1}} \leqslant \int_{1}^{b / \tau} \frac{d x}{x}=\ln \frac{b}{\tau} .
$$

This completes the proof.

Lemma 3.4. Assume that $0<a \leqslant b, k \in\{1,2, \ldots, n\}$, where $n$ is a natural number, $n \geqslant 2$. If $f:[0, a] \rightarrow \mathbb{R}$ is an absolutely continuous function obeying the condition $f(0)=0$, then an inequality holds:

$$
\int_{0}^{a} \frac{|f(t)|}{t^{n-k+1} \ln ^{2} \frac{b e}{t}} d t \leqslant \int_{0}^{a} \frac{\left|f^{\prime}(t)\right|}{t^{n-k}} d t
$$

Proof. Following the lines of the proof of Lemma 3.2 , we get:

$$
\int_{0}^{a} \frac{|f(t)| d t}{t^{n-k+1} \ln ^{2} \frac{b e}{t}} \leqslant \int_{0}^{a} \frac{d t}{t^{n-k+1} \ln ^{2} \frac{b e}{t}} \int_{0}^{t}\left|f^{\prime}(\tau)\right| d \tau=\int_{0}^{a} \frac{\mid f^{\prime}(\tau)}{\tau^{n-k}} T^{* *}(\tau) d \tau,
$$

where

$$
\begin{aligned}
T^{* *}(\tau) & =\tau^{n-k} \int_{\tau}^{a} \frac{d t}{t^{n-k+1} \ln ^{2} \frac{b e}{t}} \leqslant \tau^{n-k} \int_{\tau}^{b} \frac{d t}{t^{n-k+1} \ln ^{2} \frac{b e}{t}} \\
& =\int_{1}^{b / \tau} \frac{d x}{x^{n-k+1} \ln ^{2} \frac{b e}{\tau x}} \leqslant \int_{1}^{b / \tau} \frac{d x}{x \ln ^{2} \frac{b e}{\tau x}}=1-\frac{1}{\ln \frac{b e}{\tau}} \leqslant 1 .
\end{aligned}
$$

This completes the proof.

Proof of Theorem 3.1. We shall employ an exhausting of the domain $\Omega \neq \mathbb{R}^{n}$ by open subsets $\Omega_{j}$, formed by finitely many cubes with sides parallel to the coordinate planes. Earlier, we proposed such approach for proving some Hardy type inequality of form $(1.2)$, see, for instance, [3], [10].

Let $u \in C_{0}^{1}(\Omega)$ be a real-valued function and $u \not \equiv 0$. By $K \subset \Omega$ we denote the support of this function. In what follows we suppose that the function $u \in C_{0}^{1}(\Omega)$ is fixed. Then we construct an approximation of the domain $\Omega \subset \mathbb{R}^{n}, \Omega \neq \mathbb{R}^{n}$.

Let $h \in(0,1)$. We consider a covering of the Euclidean space $\mathbb{R}^{n}$ by the following cubes

$$
K(z, h)=\left\{x \in \mathbb{R}^{n}: x=h z+y, y \in[0, h]^{n}\right\}, \quad z \in \mathbb{Z}^{n} .
$$

Then we define a set $\Omega(h)$ as a subset $\Omega$ consisting of internal points of the union of all cubes lying in $\Omega \cap\left\{x \in \mathbb{R}^{n}:|x|<1 / h\right\}$. Then the set

$$
\Omega(h) \subset \Omega \cap\left\{x \in \mathbb{R}^{n}:|x|<1 / h\right\}
$$

consists of finitely many cubes. We suppose that the number $h \in(0,1)$ is chosen small enough so that the compact set $K$ is contained in one of the components of $\Omega(h)$.

We define a sequence $\Omega_{j}(j \in \mathbb{N})$ letting $\Omega_{j}:=\Omega\left(h_{j}\right)$, where $h_{j}=h / 2^{j-1}, j \in \mathbb{N}$. We have:

$$
\Omega=\cup_{j=1}^{\infty} \Omega_{j}, \quad K \subset \Omega_{j} \subset \Omega_{j+1} \quad(\forall j \in \mathbb{N}) .
$$


The boundary $\partial \Omega_{j}$ of an open set $\Omega_{j}$ is the union of finitely many $(n-1)$-dimensional sides of the cubes $K\left(z, h_{j}\right)$. Then we define a special partition $\Omega_{j}$ for each fixed number $j$ following paper [10].

It is obvious that the boundary $\partial \Omega_{j}$ contains cubic sides of dimension $k$ for $k \in\{0,1, \ldots, n-1\}$. Let $\bar{G}_{j m} \subset \partial \Omega_{j}$ be a $k$-dimemsional side of one of the cubes forming $\Omega_{j}$. We also mention that the singular set $S\left(\Omega_{j}\right)$ consists of the points $x \in \Omega_{j}$, for which there exist cubic sides, where $\bar{G}_{j m} \neq \bar{G}_{j m^{\prime}}$, such that

$$
P\left(x, \Omega_{j}\right) \cap \bar{G}_{j m} \neq \emptyset, \quad P\left(x, \Omega_{j}\right) \cap \bar{G}_{j m^{\prime}} \neq \emptyset .
$$

As it was pointed out in [10], the set of the points $x \in \Omega_{j}$ located at the same distance from two sides $\bar{G}_{j m} \neq \bar{G}_{j m^{\prime}}$ of dimension $k \in\{0,1, \ldots, n-1\}$ and $k^{\prime} \in\{0,1, \ldots, n-1\}$, is a bounded subset of a $(n-1)$-dimensional plane or some $(n-1)$-dimensional surface of second order.

Since the boundary set $\partial \Omega_{j}$ consists of finitely many cubic sides, then $\operatorname{mes}_{n} S\left(\Omega_{j}\right)=\operatorname{mes}_{n} \bar{S}\left(\Omega_{j}\right)=0$, where $\bar{S}\left(\Omega_{j}\right)$ stands for the closure of the set $S\left(\Omega_{j}\right)$ in the domain $\Omega_{j}$. We define the sets $G_{j m}$ as follows.

If $\operatorname{dim} G_{j m}=0$, that is, this side is a point and is one of the vertices of some cube $K\left(z, h_{j}\right)$, we let $G_{j m}:=\bar{G}_{j m}$; if $\operatorname{dim} G_{j m}=k \in\{1, \ldots, n-1\}$, then up to a rotation and translation the set $G_{j m}$ coincides with the set $(0, h)^{k}$ and $G_{j m} \subset \bar{G}_{j m}$, and the closure of $G_{j m}$ is a side $\bar{G}_{j m} \subset \partial \Omega_{j}$.

By construction, we have finitely many sets $G_{j m}, m \in\left\{1,2, \ldots, m_{j}\right\}$, such that $G_{j m} \neq G_{j m^{\prime}}$ for $m \neq m^{\prime}$ and $\partial \Omega_{j}=\cup_{m=1}^{m_{j}} G_{j m}$.

We define the following subset of $\Omega_{j}$ :

$$
W\left(G_{j m}\right)=\left\{x \in \Omega_{j} \backslash S\left(\Omega_{j}\right): P\left(x, \Omega_{j}\right)=\{y\}, y \in G_{j m}\right\} .
$$

For each continuous function $F \in C\left(\bar{\Omega}_{j}\right)$ we can use the formula

$$
\int_{\Omega_{j}} F(x) d x=\sum_{m=1}^{m_{j}} \int_{W\left(G_{j m}\right)} F(x) d x .
$$

We introduce several new notations. Let $S_{+}^{n-k}:=\left\{t \in \mathbb{R}_{+}^{n-k}:|t|=1\right\}$, where

$$
\mathbb{R}_{+}^{n-k}:=\left\{\left(t_{1}, \ldots, t_{n-k}\right) \in \mathbb{R}^{n-k}: t_{1}>0, \ldots, t_{n-k}>0\right\} .
$$

Let $G_{j m}$ be a cubic side of dimension $k \in\{0,1, \ldots, n-1\}$. Assume that $\operatorname{mes}_{n} W\left(G_{j m}\right)>0$. Up to an Euclidean motion, the set $W\left(G_{j m}\right)$ can be represented as follows:

$$
W\left(G_{j m}\right)=\left\{y+r \omega: y \in G_{j m}, \omega \in S_{+}^{n-k}, 0<r<\varphi_{k}\left(y, \omega ; G_{j m}, \Omega_{j}\right)\right\},
$$

where $\varphi_{k}$ is some continuous function satisfying the condition:

$$
0 \leqslant \varphi_{k}\left(y, \omega ; G_{j m}, \Omega_{j}\right) \leqslant \rho\left(\Omega_{j}\right) .
$$

While integrating over the set $W\left(G_{j m}\right)$, the quantity $r=\rho\left(x, \partial \Omega_{j}\right)$ will serve as one of the integration variables, and this is we shall need three types of coordinate systems: spherical coordinates, several cylindrical coordinates and a Cartesian coordinate system. Passing from multiple to reiterated integrals, we obtain several $n$ different formulae depending on the choice of the coordinate system.

We shall make use of the notations $\varphi_{k}(y, \omega)=\varphi_{k}\left(y, \omega ; G_{j m}, \Omega_{j}\right) \in\left[0, \rho\left(\Omega_{j}\right)\right]$ and of the following formulae from paper [10]:

- if $\operatorname{dim}\left(G_{j m}\right)=n-1$, then

$$
\int_{W\left(G_{j m}\right)} F(x) d x=\int_{G_{j m}} d y \int_{0}^{\varphi_{n-1}\left(y, \omega_{0}\right)} F\left(y+r \omega_{0}\right) d r
$$

- if $\operatorname{dim}\left(G_{j m}\right)=n-k, \operatorname{mes}_{n} W\left(G_{j m}\right)>0$ and $2 \leqslant k \leqslant n-1$, then

$$
\int_{W\left(G_{j m}\right)} F(x) d x=\int_{G_{j m}} d y \int_{S_{+}^{k}} d \omega \int_{0}^{\varphi_{n-k}(y, \omega)} F(y+r \omega) r^{k-1} d r ;
$$

- if $\operatorname{dim}\left(\mathrm{G}_{\mathrm{jm}}\right)=0$, i.e. $G_{j m}=\left\{x_{0}\right\}$, and $\operatorname{mes}_{n} W\left(G_{j m}\right)>0$, then

$$
\int_{W\left(G_{j m}\right)} F(x) d x=\int_{S_{+}^{n}} d \omega \int_{0}^{\varphi_{0}\left(x_{0}, \omega\right)} F\left(x_{0}+r \omega\right) r^{n-1} d r .
$$


Letting $F(x)=|u(x)| r^{-s}\left(1+r^{s} /\left((s-1) b^{s}\right)\right)$ and applying the inequality from Lemma 3.2 with the parameters

$$
a=\varphi_{n-k}(y, \omega), \quad b=\rho(\Omega) \geqslant \rho\left(\Omega_{j}\right) \geqslant a
$$

for internal integrals, we obtain the inequalities

$$
\begin{gathered}
\int_{0}^{\varphi_{n-1}\left(y, \omega_{0}\right)} \frac{|u(x)|}{r^{s}}\left(1+\frac{r^{s}}{(s-1) b^{s}}\right) d r \leqslant \frac{1}{s-n} \int_{0}^{\varphi_{n-1}\left(y, \omega_{0}\right)} \frac{\left|u_{r}^{\prime}(x)\right|}{r^{s-1}}\left(1-\frac{r^{s}}{b^{s}}\right) d r \\
\int_{0}^{\varphi_{n-k}(y, \omega)} \frac{|u(x)|}{r^{s-k+1}}\left(1+\frac{r^{s}}{(s-1) b^{s}}\right) d r \leqslant \frac{1}{s-n} \int_{0}^{\varphi_{n-k}(y, \omega)} \frac{\left|u_{r}^{\prime}(x)\right|}{r^{s-k}}\left(1-\frac{r^{s}}{b^{s}}\right) d r \\
\int_{0}^{\varphi_{0}\left(x_{0}, \omega\right)} \frac{|u(x)|}{r^{s-n+1}}\left(1+\frac{r^{s}}{(s-1) b^{s}}\right) d r \leqslant \frac{1}{s-n} \int_{0}^{\varphi_{0}\left(x_{0}, \omega\right)} \frac{\left|u_{r}^{\prime}(x)\right|}{r^{s-n}}\left(1-\frac{r^{s}}{b^{s}}\right) d r
\end{gathered}
$$

for the case $k=1,2 \leqslant k \leqslant n-1$ and $k=n$, respectively. Integrating these inequalities with respect to external variables and taking into consideration the identities $r=\rho\left(x, \partial \Omega_{j}\right)$ and

$$
\left|\frac{\partial u(x)}{\partial r}\right|=\left|\nabla u(x) \cdot \nabla \rho\left(x, \partial \Omega_{j}\right)\right|
$$

we get:

$\int_{W\left(G_{j m}\right)} \frac{|u(x)|}{\rho^{s}\left(x, \partial \Omega_{j}\right)}\left(1+\frac{\rho^{s}\left(x, \partial \Omega_{j}\right)}{(s-1) \rho^{s}\left(\Omega_{j}\right)}\right) d x \leqslant \frac{1}{s-n} \int_{W\left(G_{j m}\right)} \frac{\left|\nabla u(x) \cdot \nabla \rho\left(x, \partial \Omega_{j}\right)\right|}{\rho^{s-1}\left(x, \partial \Omega_{j}\right)}\left(1-\frac{\rho^{s}\left(x, \partial \Omega_{j}\right)}{\rho^{s}(\Omega)}\right) d x$.

Summing up these inequalities over $m \in\left\{1,2, \ldots, m_{j}\right\}$, we get:

$$
\int_{\Omega_{j}} \frac{|u(x)|}{\rho^{s}\left(x, \partial \Omega_{j}\right)}\left(1+\frac{\rho^{s}\left(x, \partial \Omega_{j}\right)}{(s-1) \rho^{s}(\Omega)}\right) d x \leqslant \frac{1}{s-n} \int_{\Omega_{j}} \frac{\left|\nabla u(x) \cdot \nabla \rho\left(x, \partial \Omega_{j}\right)\right|}{\rho^{s-1}\left(x, \partial \Omega_{j}\right)}\left(1-\frac{\rho^{s}\left(x, \partial \Omega_{j}\right)}{\rho^{s}(\Omega)}\right) d x .
$$

Finally, passing to the limit as $j \rightarrow \infty$ in this inequality and using Theorem 2.1 and Lebesgue theorem on dominated convergence, we arrive at the inequality

$$
\int_{\Omega} \frac{|u(x)|}{\rho^{s}(x, \partial \Omega)}\left(1+\frac{\rho^{s}(x, \partial \Omega)}{(s-1) \rho^{s}(\Omega)}\right) d x \leqslant \frac{1}{s-n} \int_{\Omega} \frac{|\nabla u(x) \cdot \nabla \rho(x, \partial \Omega)|}{\rho^{s-1}(x, \partial \Omega)}\left(1-\frac{\rho^{s}(x, \partial \Omega)}{\rho^{s}(\Omega)}\right) d x,
$$

which is equivalent to 3.2 . This completes the proof.

Proof of Theorem 3.2. We let

$$
\begin{aligned}
& J(u)=\frac{\rho^{-s}(\Omega)}{s-1} \int_{\Omega}|u(x)| d x, \quad c=\frac{1}{s-n}, \\
& w_{1}(x)=\frac{1}{\rho^{s}(x, \partial \Omega)}, \quad w_{2}(x)=\frac{1}{\rho^{s-1}(x, \partial \Omega)}\left(1-\frac{\rho^{s}(x, \partial \Omega)}{\rho^{s}(\Omega)}\right) .
\end{aligned}
$$

Applying the last inequality from the proof of Theorem 3.1 and Lemma 3.1, we obtain the following statement: for each real-valued function $u \in C_{0}^{1}(\Omega)$ the inequality

$$
\int_{\Omega} \frac{|u(x)|^{p}}{\rho^{s}(x, \partial \Omega)}\left(1+\frac{p \rho^{s}(x, \partial \Omega)}{(s-1) \rho^{s}(\Omega)}\right) d x \leqslant \frac{p^{p}}{(s-n)^{p}} \int_{\Omega} \frac{|\nabla u(x) \cdot \nabla \rho(x, \partial \Omega)|^{p}}{\rho^{s-p}(x, \partial \Omega)}\left(1-\frac{\rho^{s}(x, \partial \Omega)}{\rho^{s}(\Omega)}\right)^{p} d x
$$

holds. This implies inequality 3.3 of Theorem 3.2 since $p \geqslant 1, s>n$ and

$$
\left(1-\frac{\rho^{s}(x, \partial \Omega)}{\rho^{s}(\Omega)}\right)^{p} \leqslant 1-\frac{\rho^{s p}(x, \partial \Omega)}{\rho^{s p}(\Omega)}, \quad \forall x \in \Omega .
$$


The latter inequality is reduced to an elementary inequality $(1-t)^{p} \leqslant 1-t^{p}$ for $t \in[0,1]$, since

$$
0<t:=\frac{\rho^{s}(x, \partial \Omega)}{\rho^{s}(\Omega)} \leqslant 1
$$

for each point $x \in \Omega$.

Proof of Theorem 3.3. We shall need geometric constructions given in the proof of Theorem 3.1

Namely, we choose a real-valued function $u \in C_{0}^{1}(\Omega)$ with a compact support $K \subset \Omega$, construct a sequence of open sets $\Omega_{j}$ formed by the cubes such that

$$
\Omega_{j}:=\Omega\left(h_{j}\right),
$$

where $h_{j}=h / 2^{j-1}, j \in \mathbb{N}$, and

$$
\Omega=\bigcup_{j=1}^{\infty} \Omega_{j}, \quad K \subset \Omega_{j} \subset \Omega_{j+1} \quad(\forall j \in \mathbb{N}) .
$$

Then, for a fixed $j$, we consider a partition of the sets $\partial \Omega_{j}$ and $\Omega_{j}$ into subsets $G_{j m}$ and $W\left(G_{j m}\right)$. In the proof of Theorem 3.1 we obtained $n$ different formulae for the integrals over the sets $W\left(G_{j m}\right)$ depending on the choice of the coordinate system. Employing these formulae for the integrals over the sets $W\left(G_{j m}\right)$ in the case $F(x)=|u(x)| r^{-n}$ and applying the inequality from Lemma 3.3 with $a=\varphi_{n-k}(y, \omega), b=\rho(\Omega) \geqslant \rho\left(\Omega_{j}\right) \geqslant a$, for internal integrals we obtain the inequalities

$$
\begin{gathered}
\int_{0}^{\varphi_{n-1}\left(y, \omega_{0}\right)} \frac{|u(x)|}{r^{n}} d r \leqslant \int_{0}^{\varphi_{n-1}\left(y, \omega_{0}\right)} \frac{\left|u_{r}^{\prime}(x)\right|}{r^{s-1}} \ln \left(\frac{\rho(\Omega)}{r}\right) d r, \\
\int_{0}^{\varphi_{n-k}(y, \omega)} \frac{|u(x)|}{r^{n-k+1}} d r \leqslant \int_{0}^{\varphi_{n-k}(y, \omega)} \frac{\left|u_{r}^{\prime}(x)\right|}{r^{n-k}} \ln \left(\frac{\rho(\Omega)}{r}\right) d r, \\
\int_{0}^{\varphi_{0}\left(x_{0}, \omega\right)} \frac{|u(x)|}{r} d r \leqslant \int_{0}^{\varphi_{0}\left(x_{0}, \omega\right)}\left|u_{r}^{\prime}(x)\right| \ln \left(\frac{\rho(\Omega)}{r}\right) d r
\end{gathered}
$$

in the cases $k=1,2 \leqslant k \leqslant n-1, k=n$, respectively. Integrating with respect to external variables and taking into consideration identities $r=\rho\left(x, \partial \Omega_{j}\right)$ and $(3.6)$, we obtain

$$
\int_{W\left(G_{j m}\right)} \frac{|u(x)|}{\rho^{n}\left(x, \partial \Omega_{j}\right)} d x \leqslant \int_{W\left(G_{j m}\right)} \frac{\left|\nabla u(x) \cdot \nabla \rho\left(x, \partial \Omega_{j}\right)\right|}{\rho^{n-1}\left(x, \partial \Omega_{j}\right)} \ln \left(\frac{\rho(\Omega)}{\rho\left(x, \partial \Omega_{j}\right)}\right) d x .
$$

Summing up these inequalities over $m \in\left\{1,2, \ldots, m_{j}\right\}$, we get:

$$
\int_{\Omega_{j}} \frac{|u(x)|}{\rho^{n}\left(x, \partial \Omega_{j}\right)} d x \leqslant \int_{\Omega_{j}} \frac{\left|\nabla u(x) \cdot \nabla \rho\left(x, \partial \Omega_{j}\right)\right|}{\rho^{n-1}\left(x, \partial \Omega_{j}\right)} \ln \left(\frac{\rho(\Omega)}{\rho\left(x, \partial \Omega_{j}\right)}\right) d x .
$$

We pass to the limit as $j \rightarrow \infty$ in this inequality and use Theorem 2.1 and Lebesgue theorem on dominated convergence. Then we get the desired inequality (3.4) for the case $p=1$. Applying Lemma 3.1, we arrive at the inequality (3.4) for the case $p>1$. The proof is complete.

Proof of Theorem 3.4. We again use geometric constructions made in the proof of Theorem 3.1 .

We fix a real-valued function $u \in C_{0}^{1}(\Omega)$ with a compact support $K \subset \Omega$ and we construct a sequence of open sets $\Omega_{j}$ formed by the cubes. Then we employ the formulae for the integrals over the sets $W\left(G_{j m}\right)$ depending on the choice of the coordinate system for the case

$$
F(x)=|u(x)| r^{-n} \ln ^{-2} \frac{b e}{r} .
$$

We apply the inequality of Lemma 3.4 with

$$
a=\varphi_{n-k}(y, \omega), \quad b=\rho(\Omega) \geqslant \rho\left(\Omega_{j}\right) \geqslant a
$$


for the corresponding internal integrals. Then we arrive at the inequalities

$$
\begin{gathered}
\int_{0}^{\varphi_{n-1}\left(y, \omega_{0}\right)} \frac{|u(x)|}{r^{n} \ln ^{2} \frac{e \rho(\Omega)}{r}} d r \leqslant \int_{0}^{\varphi_{n-k}(y, \omega)} \frac{|u(x)|}{\varphi^{n-1}\left(y, \omega_{0}\right)} d r, \\
\int_{0}^{\varphi_{n-k}(y, \omega)} \frac{\left|u_{r}^{\prime}(x)\right|}{r^{n-k+1} \ln ^{2} \frac{e \rho(\Omega)}{r}} d r \leqslant \int_{0}^{r_{0}^{\prime}(x) \mid} \frac{\mid u_{r}^{\prime}\left(x^{n-k} d r,\right.}{\varphi_{0}\left(x_{0}, \omega\right)}\left|\int_{0}^{\left.x_{0}, \omega\right)} \frac{|u(x)|}{r \ln ^{2} \frac{e \rho(\Omega)}{r}} d r \leqslant \int_{0}^{\prime}(x)\right| d r,
\end{gathered}
$$

corresponding to the cases $k=1,2 \leqslant k \leqslant n-1, k=n$. Integrating with respect to the external variables and taking into consideration the identity $r=\rho\left(x, \partial \Omega_{j}\right)$ and (3.6), we obtain:

$$
\int_{W\left(G_{j m}\right)} \frac{|u(x)|}{\rho^{n}\left(x, \partial \Omega_{j}\right) \ln ^{2} \frac{e \rho(\Omega)}{\rho\left(x, \partial \Omega_{j}\right)}} d x \leqslant \int_{W\left(G_{j m}\right)} \frac{\left|\nabla u(x) \cdot \nabla \rho\left(x, \partial \Omega_{j}\right)\right|}{\rho^{n-1}\left(x, \partial \Omega_{j}\right)} d x .
$$

Summing up these inequalities in $m \in\left\{1,2, \ldots, m_{j}\right\}$, we get:

$$
\int_{\Omega_{j}} \frac{|u(x)|}{\rho^{n}\left(x, \partial \Omega_{j}\right) \ln ^{2} \frac{e \rho(\Omega)}{\rho\left(x, \partial \Omega_{j}\right)}} d x \leqslant \int_{\Omega_{j}} \frac{\left|\nabla u(x) \cdot \nabla \rho\left(x, \partial \Omega_{j}\right)\right|}{\rho^{n-1}\left(x, \partial \Omega_{j}\right)} d x .
$$

We pass to the limit as $j \rightarrow \infty$ in this inequality employing Theorem 2.1 and Lebesgue theorem on dominated convergence. Then we obtain desired inequality 3.5 for the case $p=1$. Applying Lemma 3.1, we arrive at inequality (3.5) for the case $p>1$. This completes the proof of the theorem.

\section{EXAmple And open PROBlem}

Let $c_{p}(s, \Omega)$ and $c_{p}^{*}(s, \Omega)$ be the constants in inequalities 1.2 and 1.3 defined by formulae 1.4 and 1.5 , respectively.

For each convex domain $\Omega \subset \mathbb{R}^{n}, \Omega \neq \mathbb{R}^{n}$, the identities

$$
c_{p}(s, \Omega)=c_{p}^{*}(s, \Omega)=\frac{(s-1)^{p}}{p^{p}}
$$

hold due to Theorem 2.2 , the estimate $c_{p}(s, \Omega) \leqslant(s-1)^{p} / p^{p}$ from paper [14] and the estimate $c_{p}^{*}(s, \Omega) \leqslant$ $c_{p}(s, \Omega)$. In particular, we have:

$$
c_{2}(2, \Omega)=c_{2}^{*}(2, \Omega)=\frac{1}{4} .
$$

Let us adduce an example, which shows that these identities are true for some non-convex domain.

We consider a circular annulus

$$
A=A(r, R)=\{z \in \mathbb{C}: r<|z|<R\}, \quad 0<r<R<\infty,
$$

with the conformal modulus $M(A)=(2 \pi)^{-1} \ln \frac{R}{r}$. By Lemma 1 in 15 and formula 3.6 , the identity holds:

$$
c_{2}(2, A)=c_{2}^{*}(2, A) \quad \text { for } \quad A=A(r, R) .
$$

In [15], the exact values of $c_{2}(2, A)$ were calculated depending on the modulus $M(A)$. It turned out that

$$
\begin{array}{r}
\lim _{M(A) \rightarrow \infty} c_{2}(2, A)=0, \quad c_{2}(2, A)=\frac{1}{4} \quad \text { for the case } M(A) \in\left(0, M^{*}\right], \\
0<c_{2}(2, A)<\frac{1}{4} \text { for the case } M(A) \in\left(M^{*}, \infty\right),
\end{array}
$$

where the critical values of the modulus is $M^{*} \approx 0.57298$.

We note that

$$
M^{*}=(2 \pi)^{-1} \ln \left(\frac{2}{q^{*}}-1\right) .
$$


The number $q^{*} \approx 0.05318$ is determined as follows. We consider the Gauss hypergeometric function

$$
F(\alpha, \beta, 1 ; \zeta)=1+\sum_{k=1}^{\infty} \frac{\zeta^{k}}{(k !)^{2}} \prod_{j=0}^{k-1}(\alpha+j)(\beta+j), \quad|\zeta|<1,
$$

and its analytic continuation in the domain $\mathbb{C} \backslash[1, \infty)$. As it was shown in [15], the formula

$$
F\left(\frac{1+i}{2}, \frac{1-i}{2}, 1 ; \zeta\right)=\frac{2 \cosh \frac{\pi}{2}}{\pi} \int_{0}^{\infty} \frac{\cos t d t}{\left(\cosh ^{2} t-\zeta\right)^{\frac{1}{2}}}, \quad \zeta \in \mathbb{C} \backslash[1, \infty),
$$

holds true and the equation

$$
X(q):=\frac{F^{\prime}\left(\frac{1+i}{2}, \frac{1-i}{2}, 1 ;-\frac{1-q}{q}\right)}{q F\left(\frac{1+i}{2}, \frac{1-i}{2}, 1 ;-\frac{1-q}{q}\right)}-\frac{F^{\prime}\left(\frac{1+i}{2}, \frac{1-i}{2}, 1 ; \frac{1-q}{2-q}\right)}{(2-q) F\left(\frac{1+i}{2}, \frac{1-i}{2}, 1 ; \frac{1-q}{2-q}\right)}=\frac{1}{1-q}
$$

has a root $q=q^{*} \in(0,1)$ such that

$$
q^{*}=\min _{q \in(0,1)}\{q: X(q)(1-q)=1 \quad \text { and } \quad X(t)(1-t) \leqslant 1 \quad \forall t \in[q, 1)\} \approx 0.05318 .
$$

It is easy to find that the critical value of the modulus $M^{*}$ corresponds to an easily remembered critical value of the quotient of the radii:

$$
c^{*}=(R / r)^{*}=\exp \left(2 \pi M^{*}\right) \approx 36.6 .
$$

Therefore, the following proposition is true.

Proposition 4.1. Let $A(r, R)=\{z \in \mathbb{C}: r<|z|<R\}$. If

$$
1<\frac{R}{r} \leqslant c^{*} \approx 36.6
$$

then $c_{2}(2, A(r, R))=c_{2}^{*}(2, A(r, R))=1 / 4$.

In conclusion we mention one of open problems.

Problem. Assume that $\Omega \subset \mathbb{R}^{2}$ is an arbitrary simply-connected domain obeying the only condition $\Omega \neq \mathbb{R}^{2}$. Whether there exists a constant $C_{2}^{*}>0$ such that the inequality

$$
\int_{\Omega}|\nabla u(x) \cdot \nabla \rho(x, \partial \Omega)|^{2} d x \geqslant C_{2}^{*} \int_{\Omega} \frac{|u(x)|^{2} d x}{\rho^{2}(x, \partial \Omega)}
$$

holds for all $u \in C_{0}^{1}(\Omega)$ ?

If the answer to the above question is positive, then the next step to obtain similar inequalities in domains $\Omega \subset \mathbb{R}^{2}$ with uniformly perfect boundaries.

\section{BIBLIOGRAPHY}

1. G.H. Hardy, J.E. Littlewood and G. Polya. Inequalities. Cambridge Univ. Press, Cambridge, (1934). [Inostr. Liter., Moscow (1948).]

2. A.A. Balinsky, W.D. Evans, R.T. Lewis. The analysis and geometry of Hardy's inequality. Springer, Heidelberg (2015).

3. F.G. Avkhadiev. Conformally invariany inequalities. Kazan, Kazan University (2020), (in Russian).

4. H. Rademacher. Über partielle und totale Differenzierbarkeit I // Math. Ann. 89:4, 340-359 (1919).

5. T.S. Motzkin. Sur quelques propriétés charactéristiques des ensembles convexes // Atti Real. Accad. Naz. Lincei Rend. Cl. Sci. Fis. Mat. Natur. Serie VI, 21, 562-567 (1935).

6. C. Mantegazza, A.C. Mennucci. Hamilton-Jacobi equations and distance functions on Riemannian manifolds // Appl. Math. Optim. 47, 1-25 (2003).

7. F.G. Avkhadiev. Properties and applications of the distance functions on open sets of the Euclidean space // Izv. VUZov. Matem. 4, 87-92 (2020). [Russian Math. (Iz. VUZ). 64:4, 78-81 (2020).] 
8. F.G. Avkhadiev. A strong form of Hardy type inequalities on domains of the euclidean space // Lobachevskii J. Math. 41:11, 2120-2135 (2020).

9. A.A. Balinsky, W.D. Evans. Some recent results on Hardy-type inequalities // Appl. Math. Inf. Sci. 4:2, 191-208 (2010).

10. F.G. Avkhadiev. Hardy-type inequalities on planar and spatial open sets // Trudy MIAN. 255, 8-18 (2006). [Proc. Steklov Inst. Math. 255, 2-12 (2006).]

11. F.G. Avkhadiev, A. Laptev. Hardy inequalities for nonconvex domains // in International Mathematical Series "Around Research of Vladimir Maz'ya. I. Function Spaces". Springer, Dordrecht. 11, 1-12 (2010).

12. F.G. Avkhadiev, K.-J. Wirths. Sharp Hardy-type inequalities with Lamb's constants // Bull. Belg. Math. Soc. Simon Stevin. 18, 723-736 (2011).

13. F.G. Avkhadiev, R.G. Nasibullin. Hardy-type inequalities in arbitrary domains with finite inner radius // Sibir. Matem. Zhurn. 55:2, 239-250 (2014). [Siberian Math. J. 55:2, 191-200 (2014).]

14. F.G. Avkhadiev, I.K. Shafigullin. Sharp estimates of Hardy constants for domains with special boundary properties // Izv. VUZov. Matem. 2, 69-73 (2014). [Russian Math. (Iz. VUZ). 58:2, $58-61(2014)$.]

15. F.G. Avkhadiev. Sharp Hardy constants for annuli // J. Math. Anal. Appl. 466:1, 936-951 (2018).

16. F.G. Avkhadiev, R.V. Makarov. Hardy type inequalities on domains with convex complement and uncertainty principle of Heisenberg // Lobachevskii J. Math. 40:9, 1250-1259 (2019).

Farit Gabidinovich Avkhadiev,

Kazan Federal University,

Kremlevskays str. 18, 420008, Kazan, Russia

E-mail: avkhadiev47@mail.ru 